Methods: Descriptive, cross-sectional study. We reviewed the medical records of outpatients with SLE (ACR 1997) who were seen since 2014 to 2016 in the Clinical Hospital of Buenos Aires, Argentina.

We evaluated sex, age, disease duration, obstetric history, use and doses of oral corticosteroids, BMI, $25 \mathrm{OH}$ vitamin D and educational level. Disease activity was scored by SLEDAI. Scores $\geq 4$ were classified as active.

The patients were classified into 2 groups, according to BMI: normal weight (NW) (19-25), overweight and obesity ( $\geq 25)$.

Results: One hundred and sixty two of 230 were evaluated. Sixty-eight patients were excluded due to lack of data. 157 (97\%) were women. Mean age for both sexes was $40.6 \pm 14.3$ years ( $p 0.70)$. Means of: SLEDAI $4.3 \pm 4.47(54.9 \%$ had SLEDAI $\geq 4)$, IMC: $27.04 \pm 5.22(56 \%$ had a BMI $\geq 25)$ and 25 -hidroxivitamin D was $25.15 \pm 9.0$.

Relation between 2 groups, according to BMI: $84.5 \%$ whom were in NW group have received steroids at some point vs $95.6 \%$ in $B M l \geq 25$ group ( $p 0.02$ ). Mean steroids doses: BMI $\geq 25$ : $9.53 \pm 10.98$ vs $5.0 \pm 7.2$ in NW group (p 0.04). Multivariate analysis showed that $\mathrm{BMI} \geq 25$ continued significantly associated with SLE duration, independently of the steroids use and other variables.

$25 \mathrm{OH}$ vitamin $\mathrm{D}$ tended to be lower in $\mathrm{BMI} \geq 25$ vs NW, but no significant differences $(24.53 \pm 9.91$ vs $25.50 \pm 9.85)(p=0.071)$

Table 1. In the multivariate analysis, Number of pregnancies was the only one variable remained significant (OR: 0.78, IC 95\%: 0.63-0.98) ( $\mathrm{p}=0.03$ )

\begin{tabular}{lccc}
\hline Variable & IMC ? 25 (n: 71) & IMC $\geq 25(\mathrm{n}: 91)$ & $\mathrm{P}$ \\
\hline Duration (months) Median (rank) & $60(1-384)$ & $84(2-480)$ & 0,02 \\
Pregnancies mean (SD) & $1,20 \pm 1,62$ & $2,64 \pm 2,84$ & 0,0 \\
Menopause (\%) & $27(38,5)$ & $51(58,6)$ & 0,009 \\
Abdominal perimeter mean (SD) & $88 \pm 8,3$ & $99 \pm 11,9$ & 0,0 \\
Depression (\%) & $9(12,5)$ & $24(26,6)$ & 0,02 \\
Chronic renal failure (\%) & $2(2,7)$ & $9(10)$ & 0,03 \\
SLEDAI $\geq 4(\%)$ & $33(45,8)$ & $56(62,2)$ & 0,02 \\
SLICC mean (SD) & $0,30 \pm 0.55$ & $1,3 \pm 1,3$ & 0,0 \\
SLICC $\geq 1(\%)$ & $18(25)$ & $64(71,1)$ & 0,0 \\
Arterial hypertension (\%) & $12(16,6)$ & $32(35,5)$ & 0,003 \\
Grade and University Studies (\%) & $32(45)$ & $19(20)$ & 0,004 \\
\hline
\end{tabular}

Conclusions: Over a half of our cohort had BMI $\geq 25$ and was characterized by older age, more frequent menopause, longer course of the disease, increased steroid use and lower educational level. Depression and hypertension were the statistically more frequent comorbidities found. Obesity was associated with more activity and accrual damage including chronic renal disease.

Disclosure of Interest: None declared

DOI: 10.1136/annrheumdis-2017-eular.2766

\section{AB0479 THE ROLE OF ANTIBODIES TO XANTHINE OXIDASE AND ADENOSINE DEAMINASE IN THE DEVELOPMENT OF ANTI-PHOSPHOLIPID SYNDROME IN PATIENTS WITH SYSTEMIC LUPUS ERYTHEMATOSUS}

A.V. Aleksandrov, I.Y. Alekhina, N.V. Aleksandrova, N.V. Nenasheva,

V.A. Aleksandrov. Federal State Budgetary Science Institution Research Institute for Clinical and Experimental Rheumatology, Volgograd, Russian Federation

Objectives: The aim of the research was to study the processes of formation of antibodies to the enzyme purine metabolism (PM) - xanthine oxidase (XO) and adenosine deaminase (ADA) - in patients with systemic lupus erythematosus (SLE) with laboratory indicants of secondary antiphospholipid syndrome (APS). Methods: 30 healthy people and 60 SLE patients with different clinical manifestations were included in this research. Antibodies to the investigated enzymes were determined in the procedure of an indirect ELISA-test using immobilized form of the corresponding enzyme as antigen array (We have developed this technique). The results of the detection of antibodies to the XO (anti-XO), antibodies to ADA (anti-ADA) and antibodies to the ADC (anti-ADC) were recorded on a spectrophotometer at a wavelength of $450 \mathrm{~nm}$. $b_{2}$-glycoprotein-I-dependent $\left(b_{2} G P-I\right)$ to the phospholipid antibodies (aPL) class lgM and lgG were determined by using a commercial test kit "Anti-Phospholipid Screen IgG/lgM" (Orgentec). The levels of $\mathrm{IgG}$ aPL/IgM did not exceed $10 \mathrm{GPL} / \mathrm{MPL}-\mathrm{U} / \mathrm{ml}$ in the group of healthy individuals. Results: According to the survey the number of SLE patients with elevated levels of anti-ADA was $51.6 \%$, the anti-XO $-53.3 \%$. There has been a number of statistically significant correlations between the presence of anti- $\mathrm{XO}$ with clinical and laboratory parameters: the level of circulating immune complexes $(r=0.297, p=0.024)$, with a hemoglobin level $(r=-0.286, p=0.042)$, the number of lymphocytes $(r=-0.29, p=0.033)$, and platelets $(r=-0,308, p=0.028)$. In $25(41.7 \%)$ patients with SLE aPL IgG class were detected, in 19 (31.7\%) - aPL IgM were detected. As a result of multivariate dispersive analysis leading role of aPL in the development of APS has been established ( $F=52,5, p<0,001)$

In positive for the presence of anti-ADA patients with SLE aPL IgG class (but not $\mathrm{aPL}$ class IgM) were detected more frequently and at higher titer than in SLE patients, without this type of antibodies $(p=0.029)$. Joint detection of anti-ADA and aPL in patients with SLE manifestations was associated with cytopenia $(p=0.019$, Fisher's exact test). It was also noted that elevated levels of anti-XO were significantly more frequently detected in patients which were also positive for the presence of aPL IgG class $(p=0.036)$ and aPL IgM class $(p=0.044)$. Comparison of the groups of patients with SLE, the positive and negative for the presence of
anti-XO, demonstrated a statistically significant increase in the frequency of signs of vasculopathy (chi-square $=4.4, p=0.042$ ).

Considering a direct link between the level of anti-XO and the level of the $\mathrm{CIC}$ we can assume that the anti-XO in the composition of the $\mathrm{CIC}$ have some impact on the transformation of "xanthine oxidase $\leftrightarrow$ xanthine dehydrogenase" in the direction of increasing the formation of $\mathrm{XO}$ and, as a consequence, a significant increase of generation of superoxide radicals, release of calcium ions into the extracellular space, and, in addition, platelet aggregation and increased blood viscosity.

Conclusions: Antibodies to enzymes PM may be a factor in the development and maintenance of vascular disorders in patients with SLE, and their detection can be used as an additional test in the complex diagnosis of SLE with symptoms of APS.

Disclosure of Interest: None declared

DOI: 10.1136/annrheumdis-2017-eular.4728

\section{AB0480 A FUNCTIONAL MAGNETIC RESONANCE IMAGING STUDY ON THE PSYCHOPATHOLOGY OF PATIENTS WITH PRIMARY SJOGREN'S SYNDROME AND ANXIETY DISORDER}

B. Cui, Y. Liu. West China Hospital, Chengdu, China

Background: Sjogren syndrome (SS) is a chronic systemic autoimmune disease characterized by exocrine gland inflammation and symptoms of oral and ocular dryness. Anxiety affects as many as $50-70 \%$ of persons living with SS. While anxiety is commonly experienced, very little is known concerning the mechanisms of cognitive dysfuction in SS.

Objectives: To reveal the psychopathology of patients with Sjogren's syndrome and anxiety disorder.

Methods: 12 patients with pSS and anxiety disorder (SAS $\geq 50), 11$ patients with pSS, and 10 healthy controls were recruited. (1) Self-rating Anxiety Scale (SAS) were used to assess anxiety level of participations. All the subjects went through functional magnetic resonance imaging (fMRI) while listening actively to neutral words, negative words and negative words alternating with neutral ones.

Results: When subjects listened to neutral words alternating with no words, prefrontal cortex and BA21 were active in patients with pSS and anxiety disorder. When subjects listened to negative words alternating with no words, patients showed increased activity in prefrontal cortex, BA21, anterior cingulate and fusiform. Furthermore, when subjects listen to negative words alternating with neutral words, patients with pSS and anxiety disorder showed more increased

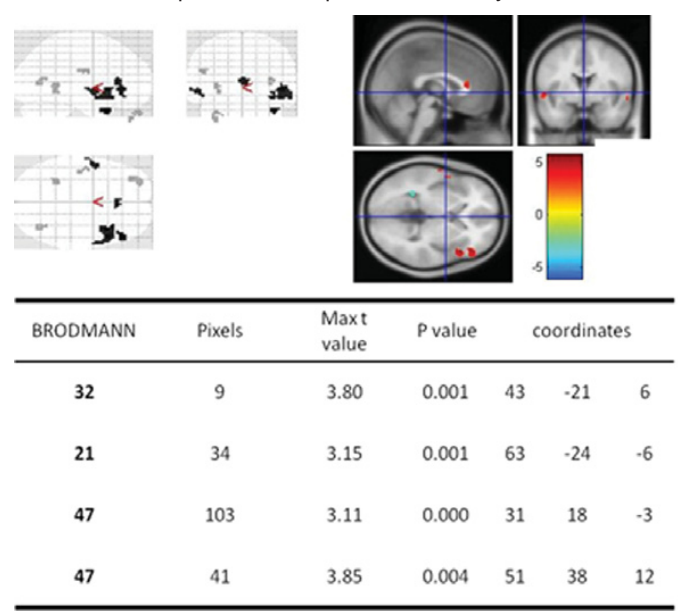

Fig.1 The results of comparison between pSS with anxiety group and pSS group, while listening to negative words alternating with neutral words. $(P<0.005)$

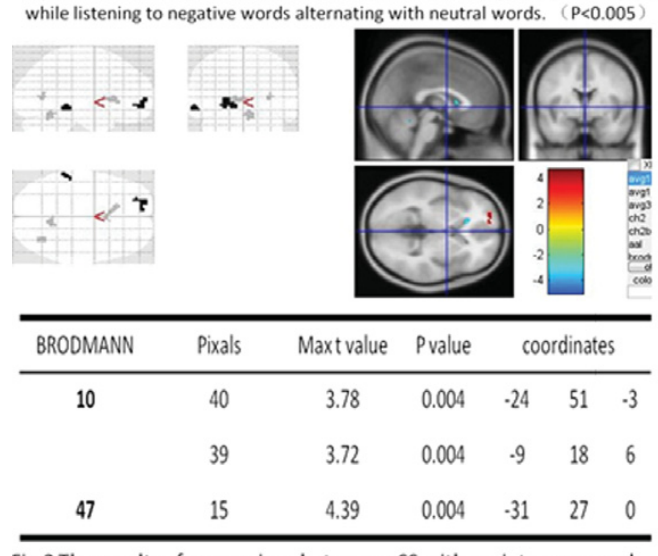

Fig. 2 The results of comparison between pSS with anxiety group and health controls, while listening to negative words alternating with neutra words. $(P<0.005$ 